\title{
-Omics approaches to help decipher molecular control of root biotic interactions in the model legume Medicago truncatula
}

\author{
C. Ben ${ }^{1 *}$, M. Toueni ${ }^{1}$, M. Rickauer ${ }^{1}$, MIRMED consortium (PIs : M. Crespi²,

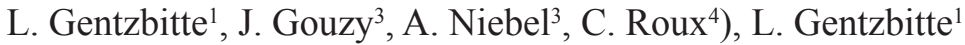 \\ ${ }^{1}$ EcoLab, Université de Toulouse, CNRS, Toulouse INP, UPS, Toulouse, France \\ ${ }^{2}$ IPS2, Orsay, France \\ ${ }^{3}$ LIPM, Toulouse, France \\ ${ }^{4}$ LRSV, Toulouse, France \\ *e-mail:ben@ensat.fr
}

Key words: transcriptomics, RNAseq, microRNA, differentially expressed genes (DEGs), gene co-expression network (WGCNA), Gene ontology (GO) enrichment, symbiosis, root diseases

Motivation and Aim: Legume roots undergo various abiotic and biotic constraints. A '-omics' high-throughput analysis was conducted to shed light on the molecular mechanisms regulating root plasticity in response to biotic stimuli in the legume model Medicago truncatula.

Methods and Algorithms: Next-generation sequencing technologies (NGS) were used to assess microRNA and gene expression in roots challenged with various symbiotic and pathogenic interactions. Differentially expressed miRNAs and genes were detected using the DESeq and edgeR packages of the R/Bioconductor statistical language. miRNA targets were predicted in silico with miRanda 3.3a customized to consider criteria available for plant miRNA targets. To identify relevant regulatory pathways involved in biotic interactions and symbiotic signals, gene co-expression network (using WGCNA R package) and Gene ontology (GO) enrichment analyses were conducted.

Results: MicroRNA regulation of the root response to different pathogenic (Verticillium alfalfae and Ralstonia solanacearum) and symbiotic (Sinorhizobium meliloti, Rhizophagus irregularis and Nod and Myc-LCO treatments) interactions were compared (MIRMED project, [1]). Specific or, conversely, common miRNAs to bacterial and fungal pathogens or symbionts have been detected. Connections in symbiotic signaling by Nod and Myc-LCO factors have also been highlighted. Regulatory network analyzes revealed co-expressed miRNAs modules that each regulate distinct sets of targets involved in different cellular functions. Molecular control of the set-up of quantitative resistance to verticillium wilt in $M$. truncatula was analyzed in more detail by transcriptomic analysis comparing the genes expressed in the roots of a resistant (A17) and susceptible (F83005.5) line at a key stage of the infective process (up to $24 \mathrm{~h}$ after inoculation) [2]. The results suggest that resistance in the A17 line of M. truncatula may be due to innate immunity combining preformed defense mechanisms and others triggered by PAMP (Pathogen Associated Molecular Patterns) and involves only a small number of induced genes. Weighted Gene-coexpression network analysis revealed five major regulatory modules in the resistant line. One module, that is significantly associated with the response to inoculation in A17, contains the majority of differentially expressed genes, and genes associated with PAMP perception and hormonal signaling as well as transcription factors. In silico analysis has shown that many of these genes also respond to other telluric pathogens in M. truncatula, suggesting a common core of transcriptional response to root pathogens.

Conclusion: The combination of '-omics' studies have revealed regulatory factors (miRNA and protein-encoding genes) potentially playing key roles in the control of pathogenic and symbiotic interactions. These candidates, which may be promising breeding targets, are undergoing functional validation by mutant analysis and genetic transformation.

Acknowledgements: This work was supported by a grant from the MIRMED project (Genoscope, CNRS). M. Toueni was supported by a doctoral grant from Mayotte department.

\section{References}

1. Formey D. et al. (2014) Diversity, conservation and plasticity of the miRNAome from Medicago truncatula roots under symbiotic and pathogenic interactions. Genome Biology. 15:457.

2. Toueni M. et al. (2016) Quantitative resistance to Verticillium wilt in Medicago truncatula involves eradication of the fungus from roots and is associated with transcriptional responses related to innate immunity. Front. Plant Sci. http:// dx.doi.org/10.3389/fpls.2016.01431 